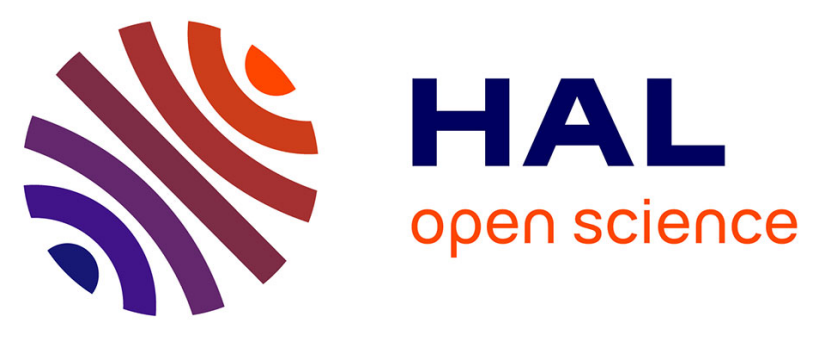

\title{
High resolution direction finding from rectangular higher order cumulant matrices: The rectangular 2Q-music algorithms
}

Hanna Becker, Pascal Chevalier, Martin Haardt

\section{- To cite this version:}

Hanna Becker, Pascal Chevalier, Martin Haardt. High resolution direction finding from rectangular higher order cumulant matrices: The rectangular 2Q-music algorithms. ICASSP 2014 - 2014 IEEE International Conference on Acoustics, Speech and Signal Processing (ICASSP), May 2014, Florence, Italy. pp.2242-2246, 10.1109/ICASSP.2014.6853998 . hal-02462474

\section{HAL Id: hal-02462474 https://hal.science/hal-02462474}

Submitted on 31 Jan 2020

HAL is a multi-disciplinary open access archive for the deposit and dissemination of scientific research documents, whether they are published or not. The documents may come from teaching and research institutions in France or abroad, or from public or private research centers.
L'archive ouverte pluridisciplinaire HAL, est destinée au dépôt et à la diffusion de documents scientifiques de niveau recherche, publiés ou non, émanant des établissements d'enseignement et de recherche français ou étrangers, des laboratoires publics ou privés. 


\title{
HIGH RESOLUTION DIRECTION FINDING FROM RECTANGULAR HIGHER ORDER CUMULANT MATRICES: THE RECTANGULAR 2Q-MUSIC ALGORITHMS
}

\author{
Hanna Becker ${ }^{(1)}$, Pascal Chevalier ${ }^{(2,3)}$ and Martin Haardt $^{(4)}$
}

(1) Université de Nice Sophia-Antipolis, CNRS, I3S, UMR7271, 06900 Sophia-Antipolis, France.

(2) CNAM, Laboratoire CEDRIC, 292 rue Saint-Martin, 75141 Paris Cédex 3, France.

(3) Thales-Communications-Security, AMS/TCP, 4 Av. des Louvresses, 92622 Gennevilliers, Cédex, France.

(4) Technische-Universität Ilmenau, Helmholtzplatz 2, PF 100565, D-98684 Ilmenau Germany

\begin{abstract}
Recently, the $2 q$-MUSIC ( $q \geq 2$ ) direction finding algorithm has been developed for non-Gaussian sources and square arrangements of the $2 q$ th-order data statistics, to overcome the main limitations of MUSIC and to improve the performance of 4-MUSIC for multiple sources. To further improve the performance of the $2 q$-MUSIC algorithm, the purpose of this paper is to extend the latter to rectangular arrangements of the data statistics, giving rise to rectangular $2 q$-MUSIC algorithms. It is shown in particular that rectangular arrangements of the higher order (HO) data statistics allow to optimize the compromise between performance and maximal number of sources to be processed. Besides, it also allows a complexity reduction for a given level of performance. These results, completely new, should open new perspectives for $\mathrm{HO}$ array processing.
\end{abstract}

Index Terms - Higher order, Virtual Array, Rectangular, Arrangements, $2 q$-MUSIC.

\section{INTRODUCTION}

Fourth-Order (FO) direction finding methods, such as 4MUSIC [1], have been developed for more than two decades for non-Gaussian sources, to overcome the limitations of second-order (SO) methods, such as MUSIC [2]. Recently, in order to still increase the performance of 4-MUSIC, the MUSIC method has been extended to an arbitrary even order $2 q(q \geq 1)$ for square arrangements of the $2 q$ th-order data statistics, giving rise to the so-called $2 q$-MUSIC algorithm [3]. It has been shown in [3] that 2q-MUSIC offers increasing performance with $q$, in terms of resolution, robustness to modelling errors and the number of sources to be processed. This performance increase is directly linked to a virtual increase of both the effective aperture and the number of sensors, $N$, of the array, introducing the $\mathrm{HO}$ virtual array (VA) concept presented in [4]. It has been proved in [4] that $2 q$-MUSIC can process up to $\mathrm{O}\left(N^{q}\right)$ sources. Furthermore, it has been shown recently in [5] that by arranging the $2 q$ th order data statistics in a $\left(N^{2 q} \mathrm{x} 1\right)$ vector, $\boldsymbol{c}_{2 q, x}$, it is possible to build a non uniform linear array of $N$ identical sensors, called $2 q$-level nested array, giving rise to a $2 q$ th order VA corresponding to a uniform linear array of $\mathrm{O}\left(N^{2 q}\right)$ virtual sensors. Using a spatial smoothing algorithm [6], it is then possible to estimate the directions of arrival (DOA) of O $\left(N^{2 q}\right)$ sources, instead of $\mathrm{O}\left(N^{q}\right)$, from the "covariance like" matrix $\boldsymbol{c}_{2 q, x} \boldsymbol{c}_{2 q, x} \mathrm{H}$, where $\mathrm{H}$ means transpose and conjugate. This result generates a more general question consisting in wondering whether it may be useful in practice to consider arbitrary rectangular arrangements of the $\mathrm{HO}$ data statistics instead of square ones for $\mathrm{HO}$ direction finding from an arbitrary array of sensors and not specific ones only. The purpose of this paper is precisely to answer this important question by extending, for arbitrary arrays of sensors, both the HO VA concept and the $2 q$-MUSIC algorithm to arbitrary rectangular arrangements of the $2 q$ th order data statistics, giving rise to rectangular $2 q$-MUSIC algorithms. It is shown in particular that rectangular arrangements of the $\mathrm{HO}$ data statistics allow to optimize the compromise between performance and maximal number of sources to be processed. Besides, it also allows a complexity reduction for a given level of performance. These results, completely new, should allow the development of new methods for HO array processing.

\section{HYPOTHESES AND DATA STATISTICS}

\subsection{Hypotheses and notations}

We consider an array of $N$ narrow-band (NB) sensors and we call $\boldsymbol{x}(t)$ the vector of complex amplitudes of the signals at the output of these sensors. Each sensor is assumed to receive the contribution of $P$ zero-mean stationary NB sources corrupted by an additive noise. We assume that the $P$ sources can be divided into $G$ groups, with $P_{g}$ sources in the group $g$, such that the sources in each group are assumed to be statistically dependent, but not perfectly coherent, while sources belonging to different 
groups are assumed to be statistically independent. Of course, $P$ is the sum of the $P_{g}$ over all the groups. Under these assumptions, the observation vector can be written as follows

$$
\boldsymbol{x}(t) \approx \sum_{p=1}^{P} s_{p}(t) \boldsymbol{a}_{p}+\boldsymbol{n}(t)=\sum_{g=1}^{G} A_{g} \boldsymbol{s}_{g}(t)+\boldsymbol{n}(t)
$$

where $\boldsymbol{n}(t)$ is the noise vector, assumed zero-mean and Gaussian and $s_{p}(t)$, independent of $\boldsymbol{n}(t)$, is the complex amplitude of source $p$. Furthermore, $A_{g}$ is the $\left(N \times P_{g}\right)$ matrix of steering vectors of sources belonging to the $g$ th group and $s_{g}(t)$ is the corresponding $\left(P_{g} \mathrm{x} 1\right)$ source vector. Without any coupling between sensors and for a plane wave propagation, component $n$ of vector $\boldsymbol{a}_{p}$ can be written as

$$
a_{p n}=\exp \left\{\mathrm{j} 2 \pi \boldsymbol{k}\left(\theta_{p}, \varphi_{p}\right)^{\mathrm{T}} \boldsymbol{p}_{n} / \lambda\right\}
$$

Here, $\lambda$ is the wavelength, $\boldsymbol{p}_{n} \triangleq\left[x_{n}, y_{n}, z_{n}\right]^{\mathrm{T}}$ is the vector of the coordinates of sensor $n, \boldsymbol{k}\left(\theta_{p}, \varphi_{p}\right) \triangleq\left[\cos \left(\theta_{p}\right) \cos \left(\varphi_{p}\right)\right.$, $\left.\sin \left(\theta_{p}\right) \cos \left(\varphi_{p}\right), \sin \left(\varphi_{p}\right)\right]^{\mathrm{T}}$ is the wave vector of source $p$, and $\left(\theta_{p}, \varphi_{p}\right)$ are the azimuth and elevation angles of source $p$.

\subsection{Statistics of the data}

The HO methods discussed in this paper exploit the information contained in the $2 q$ th order circular cumulants of the data, $\operatorname{Cum}\left[x_{i 1}(t), \ldots, x_{i q}(t), x_{i_{q+1}}(t)^{*}, \ldots, x_{i 2 q}(t)^{*}\right](1 \leq$ $\left.i_{j} \leq N\right)(1 \leq j \leq 2 q)$. The latter entries have been arranged in square matrices in [4] and [3]. These entries will be arranged in this paper in rectangular matrices giving rise, in the next sections, to the extended HO VA concept and the rectangular $2 q$-MUSIC algorithm, respectively. In situations of practical interest, the $\mathrm{HO}$ statistics of the data have to be estimated from $K$ data samples, $\boldsymbol{x}(k) \triangleq \boldsymbol{x}\left(k T_{e}\right), 1 \leq k \leq K$, where $T_{\boldsymbol{e}}$ is the sample period, using empirical estimators presented in [4] and [3].

\section{RECTANGULAR ARRANGEMENTS OF THE DATA STATISTICS}

In order to arrange the data $2 q$ th order circular cumulants into rectangular matrices, we introduce two arbitrary integers, $v$ and $l$, such that $0 \leq v \leq 2 q$ and $\sup (0, v-q) \leq l \leq \inf (v, q)$. The integer $v$ controls the size $\left(N^{v} \times N^{2 q-v}\right)$ of the rectangular $2 q$ th order cumulant matrix (as will be seen lateron, in practice only arrangements for which $v \geq q$ are of interest). The integer $l$ controls the way the data statistics are arranged in the rectangular cumulant matrix. More precisely, for given values of $q$ and $v$, let us arrange the $2 q$-uplet, $\left(i_{1}, . ., i_{q}, i_{q+1}, . ., i_{2 q}\right)$, of indices $i_{j}(1 \leq j$ $\leq 2 q)\left(1 \leq i_{j} \leq N\right)$, into one $v$-uplet and one $(2 q-v)$-uplet, indexed by $v$ and $l$, and defined by $\left(i_{1}, i_{2}, \ldots, i_{l}, i_{q+1}, \ldots\right.$, $\left.i_{q+v-l}\right)$ and $\left(i_{q+v-l+1}, \ldots, i_{2 q}, i_{l+1}, \ldots, i_{q}\right)$ respectively. As the indices $i_{j}(1 \leq j \leq 2 q)$ vary from 1 to $N$, the two latter $v$-uplet and $(2 q-v)$-uplet take $N^{v}$ and $N^{2 q-v}$ values respectively. We number, in a natural way, the $N^{v}$ values of the $v$-uplet and the $N^{2 q-v}$ values of the $(2 q-v)$-uplet by the integers $I_{v, l}$ and $J_{v, l}$ respectively, such that $1 \leq I_{v, l} \leq N^{v}$ and $1 \leq J_{v, l} \leq N^{2 q-v}$. Using the permutation invariance of the cumulants, we deduce that $\operatorname{Cum}\left[x_{i_{1}}(t), \ldots, x_{i_{i}}(t), x_{i_{q+1}}(t)^{*}, \ldots, x_{i_{2 q}}(t)^{*}\right]=$ $\operatorname{Cum}\left[x_{i 1}(t), \ldots, x_{i l}(t), x_{i_{q+1}}(t), \ldots, x_{i_{q+v-l}}(t)^{*}, x_{i_{q+v-l+1}}(t)^{*}, . .\right.$, $\left.x_{i_{2 q}}(t), \quad x_{i_{l+1}}(t), . ., \quad x_{i_{q}}(t)\right]$, where $*$ means complex conjugate. Assuming that this term is the element $\left[I_{v l}, J_{v, l}\right]$ of the rectangular cumulant matrix denoted as $C_{2 q, x}^{(v, l)}$, it is easy to verify, from section 2.1 and for a Gaussian noise, that the $\left(N^{v} \times N^{2 q-v}\right) C_{2 q, x}^{(v, l)}$ matrix can be written as

$$
\begin{aligned}
C_{2 q, x}^{(v, l)}= & \sum_{g=1}^{G}\left[A_{g}^{\otimes l} \otimes A_{g}{ }^{* \otimes(v-l)}\right] C_{2 q, s g}^{(v, l)}\left[A_{g}^{\otimes(q-v+l)}\right. \\
& \left.\otimes A_{g}{ }^{* \otimes(q-l)}\right]^{\mathrm{H}}+\eta_{2} V(v, l) \delta(q-1) .
\end{aligned}
$$

Here, $C_{2 q, s g}^{(v, l)}$ is the $\left(P_{g}{ }^{v} \times P_{g}^{2 q-v}\right)$ matrix of the $2 q$ th order circular cumulants of $s_{g}(t), \eta_{2}$ is the mean power of the noise per sensor, $V(v, l)$, defined for $q=1$ only, is the $\left(N^{v} \mathrm{x}\right.$ $\left.N^{2-v}\right)(0 \leq v \leq 2)$ normalized rectangular spatial coherence matrix of the noise such that the total input power of the noise is $N \eta_{2}, \delta($.$) is the Kronecker symbol, \otimes$ is the Kronecker product and $A_{g}{ }^{\otimes l}$ is the $\left(N^{l} \mathrm{x} P_{g}^{l}\right)$ matrix defined by $A_{g} \otimes l \triangleq A_{g} \otimes A_{g} \otimes \ldots . . \otimes A_{g}$ with a number of Kronecker product equal to $l-1$. In particular, for $v=q, C_{2 q, x}^{(v, l)}$, reduces to the $\left(N^{q} \times N^{q}\right)$ square matrix $C_{2 q, x}^{(q, l)}$, used in [3] where it is denoted by $C_{2 q, x}(l)$.

\section{RECTANGULAR 2Q-MUSIC ALGORITHMS}

\subsection{Hypotheses}

To develop the rectangular $2 q$-MUSIC algorithms for the arrangement $C_{2 q, x}^{(v, l)}$, we introduce some hypotheses:

H1 : $P_{g}<N, 1 \leq g \leq G$

$\mathrm{H} 2: A_{g}{ }^{\otimes} \otimes A_{g}{ }^{* \otimes(v-l)}$ and $A_{g}{ }^{\otimes(q-v+l)} \otimes A_{g}{ }^{* \otimes(q-l)}$ have a rank equal to $P_{g} \operatorname{Min}(v, 2 q-v), 1 \leq g \leq G$

$$
\begin{aligned}
& \mathrm{H} 3: P(G, q, v) \triangleq \sum_{g=1}^{G} P_{g} \operatorname{Min}(v, 2 q-v)<N^{\operatorname{Min}(v, 2 q-v)} \\
& \left.\mathrm{H} 4: \bar{A}_{q,}^{(v, l) \triangleq\left[A_{1} \otimes l_{\otimes} A_{1}{ }^{*} \otimes(v-l)\right.}, . ., A_{G}{ }^{\otimes l} \otimes A_{G}{ }^{*} \otimes(v-l)\right] \text { and }
\end{aligned}
$$
have $\operatorname{rank} P(G, q, v)$

In particular, for statistically independent sources $(G=P)$, $P(G, q, v)=P, A_{g}$ reduces to $\boldsymbol{a}_{g}$ and $\mathrm{H} 1$ to $\mathrm{H} 4$ reduce to

$$
\text { H1': } P<N^{\operatorname{Min}(v, 2 q-v)}
$$

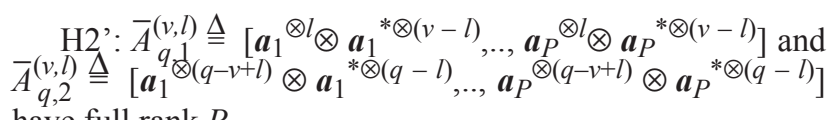
have full rank $P$

\subsection{Rectangular 2q-MUSIC algorithm}

The matrix $C_{2 q, s g}^{(v, l)}$ has full rank, $P_{g} \operatorname{Min}(v, 2 q-v)$, in general since the components of $\boldsymbol{s}_{g}(t)$ are statistically dependent. We then deduce from $\mathrm{H} 1$ to $\mathrm{H} 4$ that, for $q>1, C_{2 q, x}^{(v, l)}$ has a rank equal to $P(G, q, v)$. To build a MUSIC-like algorithm 
from $C_{2 q, x}^{(v, l)}$ for $q>1$, we first compute the singular value decomposition (SVD) of the latter, given by

$$
C_{2 q, x}^{(v, l)}=\left[U_{2 q, s}^{(v, l)} U_{2 q, n}^{(v, l)}\right]\left[\begin{array}{ll}
\Sigma_{2 q, s}^{(v, l)} & \mathrm{O}_{2 q, 1}^{(v, l)} \\
\mathrm{O}_{2 q, 2}^{(v, l)} & \Sigma_{2 q, n}^{(v, l)}
\end{array}\right]\left[\begin{array}{c}
V_{2 q, s}^{(v, l) \mathrm{H}} \\
\\
V_{2 q, n}^{(v, l) \mathrm{H}}
\end{array}\right]
$$

where $\Sigma_{2 q, s}^{(v, l)}$ is the $(P(G, q, v) \times P(G, q, v))$ diagonal matrix of the nonzero singular values of $C_{2 q, x}^{(v, l)}, U_{2 q, s}^{(v, l)}$ and $V_{2 q, s}^{(v, l)}$ are the $\left(N^{v} \times P(G, q, v)\right)$ and $\left(N^{2 q-v} \times P(G, q, v)\right)$ unitary matrices of the left and right singular vectors of $C_{2 q, x}^{(v, l)}$ associated with the $P(G, q, v)$ non zero singular values respectively, $\mathrm{O}_{2 q, 1}^{(v, l)}$ and $\mathrm{O}_{2 q, 2}^{(v, t)}$ are the $\left(P(G, q, v) \times\left(N^{2 q-v}-\right.\right.$ $P(G, q, v)))$ and the $\left(\left(N^{v}-P(G, q, v)\right) \times P(G, q, v)\right)$ null matrices respectively, $\Sigma_{2 q, n}^{(v, l)}$ is the $\left(\left(N^{v}-P(G, q, v)\right) \times\left(N^{2 q-v}\right.\right.$ - $P(G, q, v)))$ diagonal matrix of the zero singular values of $C_{2 q}^{(v, l)}, U_{2 q, \eta}^{(v, l)}$ and $V_{2 q, n}^{(v, l)}$, such that $U_{2 q, s}^{(v, l)} \mathrm{H}_{2 q, n}^{(v, l)}=\mathrm{O}_{2 q, 2}^{(v, l)}$ and $V_{2 q, s}^{(v, l)} \mathrm{H}_{2 q, n}^{(v, l)}=\mathrm{O}_{2 q, 1}^{(v, l, n}$, are the $\left(N^{v} \times\left(N^{v}-P(G, q, v)\right)\right)$ and $\left(N^{2 q-v} \times\left(N^{2 q-v}-P(G, q, v)\right)\right)$ unitary matrices of the left and right singular vectors of $C_{2 q, x}^{(v, l)}$ associated with the zero singular values. As $\operatorname{Span}\left\{U_{2 q, s}^{(v, l)}\right\}=\operatorname{Span}\left\{\bar{A}_{q, 1}^{(v, l)}\right\}$, we deduce that all the columns of all the matrices $A_{g} \otimes l^{q} \otimes A_{g}{ }^{*} \otimes(v-l), 1 \leq$ $g \leq G$, are orthogonal to all the columns of $U_{2 q, n}^{(v, l)}$. Let $\boldsymbol{a}_{i g}$ be the steering vector of the $i$ th source in the gth group. Then the vector $\boldsymbol{a}_{i g} \otimes l^{\otimes} \otimes \boldsymbol{a}_{i g}{ }^{* \otimes(v-l)}$ corresponds to one column of $A_{g} \otimes l_{\otimes} A_{g}{ }^{* \otimes(v-l)}$. Hence, all vectors $\left\{\boldsymbol{a}_{i g}{ }^{\otimes l} \otimes \boldsymbol{a}_{i g}{ }^{* \otimes(v-l)}, 1\right.$ $\left.\leq i \leq P_{g}, 1 \leq g \leq G\right\}$ are orthogonal to the columns of $U_{2 q, n}^{(v, l)}$ and are solutions of the following equation

$$
\left[\boldsymbol{a}^{\otimes l} \otimes \boldsymbol{a}^{* \otimes(v-l)}\right]^{\mathrm{H}} U_{2 q, n}^{(v, l)} U_{2 q, n}^{(v, l) \mathrm{H}}\left[\boldsymbol{a}^{\otimes l} \otimes \boldsymbol{a}^{* \otimes(v-l)}\right]=0
$$

which corresponds to the heart of the rectangular $2 q$ MUSIC algorithm for the arrangement $C_{2 q, x}^{(v, l)}$, called $2 q-$ $\operatorname{MUSIC}(v, l)$. In practice, $U_{2 q, n}^{(v, l)}$ has to be estimated from the observations and the DOAs of the sources may be found by searching for the minima of the left hand side of (5).

\section{PROPERTIES AND PERFORMANCE OF RECTANGULAR 2Q-MUSIC ALGORITHMS}

\subsection{Hypotheses}

The best properties and performance of $2 q$-MUSIC $(v, l)$, are obtained for statistically independent sources [3], which are considered in section 5, and for which (3) reduces to

$$
C_{2 q, x}^{(v, l)}=\sum_{p=1}^{P} c_{2 q, s_{p}} \boldsymbol{a}_{q, p, 1}^{(v, l)} \boldsymbol{a}_{q, p, 2}^{(v, l) \mathrm{H}}+\eta_{2} V(v, l) \delta(q-1)
$$

Here, $c_{2 q, s_{p}} \triangleq \mathrm{Cum}\left[s_{i_{1}}(t), \ldots, s_{i_{q}}(t), s_{i_{q+1}}(t)^{*}, \ldots, s_{i_{2 q}}(t)^{*}\right]$, with $i_{j}=p(1 \leq j \leq 2 q)$, is the $2 q$ th order circular

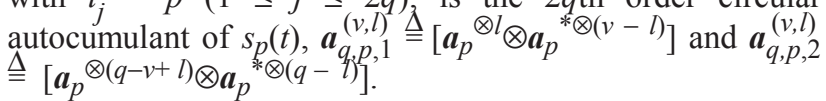

\subsection{Performance of $2 q$-MUSIC algorithms}

We deduce from (6) that each source $p$ contributes to $C_{2 q, x}^{(v, l)}$ through a rank one matrix $c_{2 q, s_{p}} \boldsymbol{a}_{q, p, 1}^{(v, l)} \boldsymbol{a}_{q, p, 2}^{(v, l) \mathrm{H}}$ whose left and right vectors, $\boldsymbol{a}_{q, p, 1}^{(v, l)}$ and $\boldsymbol{a}_{q, p, 2}^{(v, l)}$, correspond to the $\left(N^{v} \times 1\right)$ left and $\left(N^{2 q-v} \times 1\right)$ right virtual steering vectors of source $p$ for the considered array of sensors. It has been shown in [4] that $\boldsymbol{a}_{q, p, 1}^{(v, l)}$ and $\boldsymbol{a}_{q, p, 2}^{(v, l)}$ can be considered as true steering vectors of the source $p$ but for two VA of $N^{v}$ and $N^{2 q-v}$ virtual sensors (VS), called hereafter left and right VA respectively. The positions of the left and right VS are defined respectively by

$$
\sum_{j=1}^{l} \boldsymbol{p}_{k j}-\sum_{u=1}^{v-l} \boldsymbol{p}_{k l+u} \text { and } \sum_{j=1}^{q-v+l} \boldsymbol{p}_{k j}-\sum_{u=1}^{q-l} \boldsymbol{p}_{k q-v+l+u}
$$

where $1 \leq k_{j} \leq N$ for $1 \leq j \leq j_{\max }$ with $j_{\max }=v$ and $j_{\max }=$ $2 q-v$ for the left and right VA respectively. These concepts of left and right VA extend to rectangular arrangements $C_{2 q, x}^{(v, l)}$ the HO VA concept introduced in [4] for square arrangements $C_{2 q, x}^{(q, l)}$ for which the left and right virtual steering vectors and VA coincide. As $2 q$-MUSIC $(v, l)$ aims at identifying the vectors $\boldsymbol{a}_{q, p, 1}^{(v, l)}, 1 \leq p \leq P$, from the DOA estimates of the sources, the performance of $2 q$-MUSIC( $v$, l) for multiple sources is directly controlled by the left VA associated with $C_{2 q, x}^{(v, l)}$. This result generates a consequence enlightening the interest of rectangular arrangements with respect to square ones. Indeed, considering two integers $q_{1}$ and $q_{2}$ such that $1 \leq q_{2}<q_{1} \leq 2 q_{2}$ and choosing $l$ such that $q_{1}-q_{2} \leq l \leq q_{2}$, it is straightforward to verify that $\boldsymbol{a}_{q_{2}, p, 1}^{\left(q_{1}, l\right)}$ and $\boldsymbol{a}_{q_{1}, p, 1}^{\left(q_{1}, l\right)}$ exist and coincide. This means that the left VA associated with $C_{2 q_{2}, x}^{\left(q_{1}, l\right)}$ and $C_{2 q_{1}, x}^{\left(q_{1}, l\right)}$ also coincide. This result proves that using a rectangular arrangement, $C_{2 q_{2}, x}^{\left(q_{1}, l\right)}$, of the $2 q_{2}$ th order circular cumulants of the data allows to achieve the same performance, for multiple sources scenarios, in terms of potential resolution and robustness to modelling errors, as using a square arrangement, $C_{2 q_{1}, x}^{\left(q_{1}, l\right)}$, of circular cumulants of the data with an order $2 q_{1}$ higher than $2 q_{2}$. However, for given performance, the rectangular arrangement $C_{2 q_{2}, x}^{\left(q_{1}, l\right)}$ generates both a complexity reduction and a lower variance in the statistics estimate than the square arrangement $C_{2 q_{1}, x}^{\left(q_{1}, l\right)}$, hence an overall best global performance. This result, which will be illustrated in section 5.5 for $\left(q_{1}, q_{2}, l\right)=(3,2,1)$ and $(3,2,2)$, proves that for a given statistics order $2 q$, the parameter $v>q$ of the rectangular arrangement $C_{2 q, x}^{(v, l)}$ indicates the statistics order, $2 v>2 q$, of the square arrangement, $C_{2 v, x}^{(v, l)}$, whose performance, in terms of potential resolution and robustness to modelling errors, can be achieved. This result proves in particular the increasing performance with $v$ of $2 q$ $\operatorname{MUSIC}(v, l)$ as long as $q<v \leq 2 q-1$ to be able to process multiple sources from an arbitrary array of sensors.

\subsection{Identifiability of $2 q$-MUSIC algorithms}

Denoting by $r_{2 q, x}^{(v, l)}$ the maximal rank of $C_{2 q, x}^{(v, l)}$ in the absence of noise, it is straightforward to show that the maximal number of sources which may be processed by $2 q$ $\operatorname{MUSIC}(v, l)$ is equal to $r_{2 q, x}^{(v, l)}-1$. Denoting by $N_{2 q, 1}^{(v, l)}$ and $N_{2 q, 2}^{(v, l)}$ the number of different VS of the left and right $2 q$ th 
order VA associated with $C_{2 q, x}^{(v, l)}$ respectively, it is obvious from (6) that $r_{2 q, x}^{(v, l)}=\operatorname{Min}\left(N_{2 q, 1}^{(v, l)}, N_{2 q, 2}^{(v, l)}\right.$. For given values of $(q, l), r_{2 q, x}^{(v, l)}$ is then maximized for $v=q$, i.e., by the square arrangement $C_{2 q, x}^{(q, l)}$ for which $r_{2 q, x}^{(q, l)}=N_{2 q, 1}^{(q, l)}=N_{2 q, 2}^{(q, l)}$, denoted by $N_{2 q}^{q}$ in [4]. Consequently, for given values of $q$ and $l$, as the performance of $2 q$-MUSIC $(v, l)$ for multiple sources increases with $v$, as shown in section 5.2, in practice $v$ should be chosen such that $q \leq v \leq 2 q$ to optimize the performance for a given number of sources (greater than one) to be processed. This means that $C_{2 q, x}^{(v, l)}$ must be either square or tall and in this case, $r_{2 q, x}^{(v, l)}=N_{2 q, 2}^{(v, l)}$. As $N_{2 q, 2}^{(v, l)}$ decreases with $v$ while the performance improves, there is a trade off between the number of sources to be processed and the performance for multiple sources and a compromise has to be found in practice. The possibility to adjust the compromise between the number of sources to be processed by $2 q$-MUSIC $(v, l)$ and its performance for multiple sources corresponds to one of the main interests of rectangular arrangements with respect to square ones.

\subsection{Optimal arrangement index $l$}

Finally, for given values of $q$ and $v \geq q$, and from an asymptotical (as $K \rightarrow \infty$ ) performance point of view, the index $l$ is of no importance for the left VA associated with $C_{2 q, x}^{(v, l)}$ as shown in [4]. Thus, as $K$ becomes large, the optimal index $l$ is the one which maximizes the number of sources to be processed, i.e. $N_{2 q, 2}^{(v, l)}$. Using the results of [4], we deduce that $l_{\mathrm{opt}}=v / 2$ if $v$ is even and $l_{\mathrm{opt}}=(v-1) / 2$ if $v$ is odd.

\subsection{Computer simulations}

The results of this paper are illustrated in this section by computer simulations. Two performance criteria presented in [3] are considered for each source. The first one is the probability of non-aberrant results, i.e., the probability that the estimated left hand side of (5) is lower than a threshold $\eta$. The second one is the averaged root mean square error (RMSE), computed from the non-aberrant results. We assume that 2 synchronized statistically independent QPSK sources, sampled at the symbol rate, are received by a Uniform Circular Array (UCA) of $N=3$ omnidirectional sensors with a radius $r$ such that $r=0.3 \lambda$. The 2 QPSK sources have the same input SNR equal to $10 \mathrm{~dB}$ and a direction of arrival equal to $\theta_{1}=90^{\circ}$ and $\theta_{2}$ respectively. Under these assumptions, Figure 1 shows the variations, as a function of the number of snapshots $K$, of the RMSE for the source $1, \mathrm{RMSE}_{1}$, (we obtain similar results for the source $2)$, estimated from $M=500$ realizations, at the output of 2 $\operatorname{MUSIC}(1,1), 4-\operatorname{MUSIC}(2,1), 4-\operatorname{MUSIC}(2,2), 4-\operatorname{MUSIC}(3$, 1), 4-MUSIC(3, 2), 6-MUSIC(3, 1) and 6-MUSIC(3, 2). For these figures, $\theta_{2}=105^{\circ}$ and the steering vectors $\boldsymbol{a}_{p}(1 \leq p \leq$ 2 ) are corrupted by a zero-mean circular Gaussian modelling error vector $\boldsymbol{e}_{p}$, such that $\mathrm{E}\left[\boldsymbol{e}_{p} \boldsymbol{e}_{S} \mathrm{H}\right]=\sigma^{2} \delta_{p s} \mathrm{I}_{N}$ where $\sigma=$ 0.03 . Note that beyond $K=100$ snapshots, the probability of non-aberrant results with $\eta=0.1$ is equal to 1 for all the methods. Note, above a few hundred of snapshots, the better performance of the rectangular 4-MUSIC algorithms, 4$\operatorname{MUSIC}(3,1)$ and 4-MUSIC $(3,2)$, with respect to the square ones, 4-MUSiC(2, 1) and 4-MUSiC $(2,2)$. Note also in this case the almost same performance of rectangular 4-MUSIC algorithms with the square 6-MUSIC algorithms. To complete the previous results, we consider again the previous scenario with modelling errors but with now an infinite number of snapshots $K$ and an arbitrary value of $\Delta \theta$. Under these assumptions, Figure 2 shows the RMSE 1 as a function of $\Delta \theta$ at the output of the previous methods. We note that rectangular 4-MUSIC $(3,1)$ and $4-\operatorname{MUSIC}(3,2)$ outperform square 4-MUSIC(2, 1) and 4-MUSIC(2, 2) especially for close sources and allow to obtain the performance of square 6-MUSIC $(3,1)$ and 6-MUSIC $(3,2)$ using $4^{\text {th }}$ order statistics only.

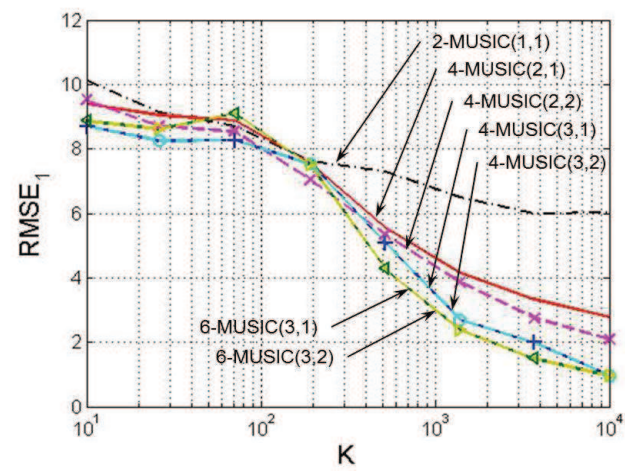

Figure 1-RMSE of the source $1\left(^{\circ}\right)$ as a function of $K, P=2, N$ $=3, U C A, S N R=10 \mathrm{~dB}, \Delta \theta=15^{\circ}, \sigma=0.03$.

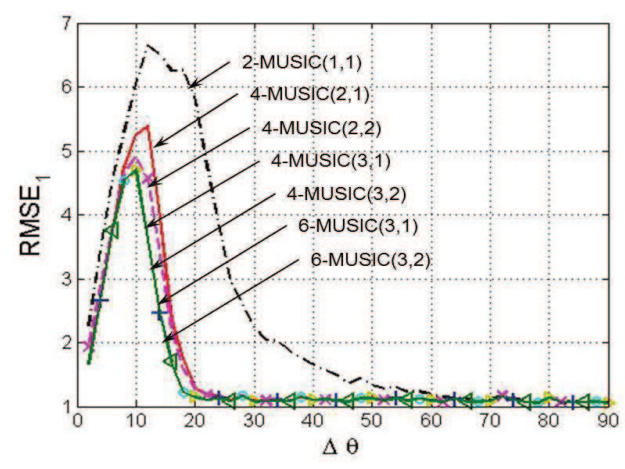

Figure $2-R M S E$ of the source $1\left(^{\circ}\right)$ as a function of $\Delta \theta^{\circ}, P=2$, $N=3, U C A, S N R=10 d B, K=\infty, \sigma=0.03$.

\section{CONCLUSION}

In this paper, rectangular arrangements of the $\mathrm{HO}$ data statistics have been considered for direction finding with the proposed rectangular $2 q$-MUSIC algorithms. It has been shown that these rectangular arrangements allow to optimize the compromise between performance and maximal number of sources to be processed. Besides, they also allow a complexity reduction for a given level of performance. These results, completely new, should allow the development of new methods for HO array processing. 


\section{REFERENCES}

[1] B. Porat, B. Friedlander, "Direction finding algorithms based on higher order statistics", IEEE Trans. Signal Processing, Vol 39, N9, pp. 2016-2024, Sept 1991.

[2] R.O. Schmidt, "Multiple emitter location and signal parameter estimation", IEEE Trans. Ant. Prop., Vol 34, N³, pp. 276-280, March 1986.

[3] P. Chevalier, A. Ferréol, L. Albera, "High resolution direction finding from higher order statistics : the $2 q$-MUSIC algorithm", IEEE Trans. Signal Processing, Vol 54, Nº, pp. 2986-2997, Aug. 2006

[4] P. Chevalier, L. Albera, A. Ferréol, P. Comon, "On the Virtual Array concept for higher order array processing", IEEE Trans. Signal Processing, Vol 53, N4, pp. 1254-1271, April 2005.

[5] P. Pal, P.P. Vaidyanathan, "Multiple Level Nested Array: An Efficient Geometry for 2qth Order Cumulant Based Array Processing ", IEEE Trans. Signal Processing, Vol 60, N³, pp. 1253-1269, March 2012.

[6] P. Pal, P.P. Vaidyanathan, "Nested Arrays: A Novel approach to array processing with enhanced degrees of freedom", IEEE Trans. Signal Processing, Vol 58, Nº, pp. 4167-4181, Aug. 2010.

$N B$ : Note that this work has been done at Thales Communications and Security during the Master 2 research project of Hanna Becker. 